ARTIG0

Recebido em: 28/04/2017

Aceito em: 09/04/2018

\title{
Práticas do pensamento enxuto para a gestão estratégica da informação e do conhecimento
}

\author{
Lean thinking practices for the information and knowledge \\ strategic management
}

\section{Resumo}

A aplicação dos princípios do Pensamento Enxuto (Lean Thinking) determina a estrutura e os processos nas organizações que, mediante a eliminação de desperdícios e iniciativas de melhoria contínua, buscam obter melhores níveis de produtividade e eficiência. Nesse cenário, os processos de gestão da informação e do conhecimento organizacional são relevantes, visto que o reconhecimento da importância estratégica da informação e a disseminação do conhecimento entre as diferentes funções da organização são fatores críticos para o alcance de desempenho superior. Assim, o objetivo deste estudo é identificar que práticas do Pensamento Enxuto são fatores facilitadores para a criação e disseminação de conhecimento organizacional. Trata-se de uma pesquisa com base em uma revisão bibliográfica da produção científica desenvolvida no período de 2007 a 2016 sobre estudos que abordaram aspectos da gestão de conhecimento organizacional em contextos de aplicação dos princípios do Pensamento Enxuto, depositados nas bases de dados Scopus, Lista-Ebsco, Emerald, Web of Science e Science Direct. O método adotado é o PRISMA (Preferred Reporting Items for Systematic Reviews). A revisão de literatura evidenciou a existência das seguintes práticas do Pensamento Enxuto, em suas aplicações, que facilitam a criação e disseminação de conhecimento organizacional: estrutura organizacional, autonomia, mecanismos de integração, replicação de conhecimento, ambidesteridade contextual, aprendizagem e uso de ferramentas Lean. As características identificadas nestas práticas tornam possíveis todas as fases do processo de conversão de conhecimento (processo SECI) e estimulam redes de interações nas quais o conhecimento é criado, compartilhado e utilizado pelas pessoas nos ambientes organizacionais. Ainda se concluiu que a aplicação dos princípios do Pensamento Enxuto, via de regra, possibilita ganhos de eficiência e produtividade, que são auferidos pela eliminação de desperdícios nos processos de trabalho e que promove o desenvolvimento de um contexto adequado para a gestão do conhecimento organizacional.

Palavras-chave: Gestão Estratégica. Gestão da Informação. Gestão do Conhecimento. Criação de Conhecimento. Pensamento Enxuto. v. 23 , n. esp., 2018 p. 76-89

ISSN 1518-2924 


\begin{abstract}
The deployment of the Lean Thinking principles determines structure and processes in organizations which seek to achieve better levels of productivity and efficiency by eliminating waste and continuous improvement initiatives. The processes of information management and organizational knowledge management are relevant in this scenario, since the recognition of the strategic importance of information and the dissemination of knowledge among the different functions of the organization are critical factors for the achievement of a superior performance. Thus, the purpose of this study is to identify that Lean Thinking practices are facilitating factors for a creation and dissemination of organizational knowledge. It is a research based on a literature review of the scientific production developed from 2007 to 2016 on studies that investigated aspects of organizational knowledge management in contexts of Lean Thinking deployment available in the Scopus, Lista-Ebsco, Emerald, Web of Science and Science Direct databases. The method chosen was PRISMA (Preferred Reporting Items for Systematic Reviews). The literature review evidenced the existence of Lean Thinking practices that facilitate the creation and dissemination of organizational knowledge, such as: organizational structure, autonomy, integration mechanisms, knowledge replication, contextual ambidexterity, learning and use of lean tools. The characteristics identified in these practices make possible all phases of the knowledge conversion process (SECI process) and promote networks of interactions in which knowledge is created, shared and used by people in organizational environments. It is also concluded that the deployment of the Lean Thinking principles allows gains in efficiency and productivity that are obtained by the elimination of wastes in the work processes and that promotes the development of a suitable context for the management of organizational knowledge.
\end{abstract}

Keywords: Strategic Management. Information Management. Knowledge Management. Knowledge Creation. Lean Thinking.

\title{
1 INTRODUÇÃO
}

No processo de gestão do conhecimento organizacional, a interação dos indivíduos e o desenvolvimento de um contexto fecundo para a criação e disseminação de conhecimento são fatores primordiais (VON KROGH; ICHIJO; NONAKA, 2001). Na perspectiva de Choo (2003), a informação desempenha uma função estratégica no crescimento e na capacidade de adaptação das organizações, seja na interpretação do ambiente externo, na criação, organização e processamento da informação para a obtenção de novos conhecimentos e, também nos processos de tomada de decisão.

Cavalcante e Valentim (2010) afirmam que a informação e o conhecimento permeiam praticamente todos os processos empresariais e constituem recursos estratégicos para o desenvolvimento das organizações; todavia, a informação somente será efetiva quando o seu valor for percebido pelos indivíduos e quando prevalecer uma cultura de compartilhamento da informação no contexto organizacional.

Womack e Jones (1998) desenvolveram a abordagem do Pensamento Enxuto (Lean Thinking) em decorrência da Produção Enxuta (Lean Production), estendendo a aplicação de seus conceitos e ferramentas para além dos ambientes de manufatura, como uma filosofia de gestão centrada na eliminação de desperdícios e na busca da máxima eficiência nos processos de trabalho.

Dombrowski, Mielke e Engel (2012) argumentam que a estrutura organizacional e a gestão do conhecimento estão inter-relacionadas nos sistemas de produção enxuta, onde o conhecimento é transferido entre as diferentes funções da organização e a sua aplicação afeta todos os indivíduos, em todos os níveis da organização.

Considerando a prevalência dessa inter-relação entre a estrutura organizacional e a gestão do conhecimento nos ambientes em que são aplicados os princípios do Pensamento Enxuto, questiona-se que práticas dessa abordagem favorecem a interação social e o desenvolvimento de um contexto adequado para a criação e disseminação de conhecimento. Portanto, esta pesquisa objetiva identificar as práticas do Pensamento 
Enxuto que são fatores facilitadores para a criação e disseminação de conhecimento organizacional.

\section{LEAN THINKING E A CRIAÇÃO DE CONHECIMENTO ORGANIZACIONAL}

O conceito Enxuto (Lean) surgiu no contexto de estudos do Massachusetts Institute of Technology (MIT) sobre a indústria automobilística mundial, no período de 1985 a 1990, para descrever o Sistema Toyota de Produção (STP), um sistema de produção de automóveis desenvolvido pela empresa japonesa Toyota após a Segunda Guerra Mundial. Este sistema, também conhecido como Produção Enxuta, revolucionou a indústria automobilística e disseminou uma nova prática para os processos produtivos, com o objetivo de alcançar melhores níveis de produtividade e eficiência por meio da eliminação de todo e qualquer tipo de desperdício e a implantação de melhorias contínuas nos processos produtivos (WOMACK; JONES; ROOS, 1992).

O surgimento da Produção Enxuta se deu no período de 1949-1950 e seus fundadores foram Eiji Toyoda e Thaiichi Ohno. Ohno, ao visitar as instalações da Ford em Detroit, nos Estados Unidos da América, constatou que o processo de produção em massa era cheio de desperdícios. Então, começou a desenvolver ações para combater os desperdícios no processo produtivo da empresa Toyota. Suas iniciativas são consideradas como os passos primordiais para a implementação da Produção Enxuta, e abrangem várias ações, tais como: (1) Agrupar os trabalhadores em equipes; (2) Tornar as equipes aptas a desempenhar múltiplas atividades; (3) Estabelecer sistemática para as equipes proporem melhorias nos processos produtivos; (4) Dar autonomia para os trabalhadores interromperem a produção quando da ocorrência de problemas e buscarem, em equipe, a solução. Como resultados dessas ações, a Toyota verificou a diminuição drástica do índice de reparos, a elevação dos níveis de qualidade dos produtos acabados, a prevalência da colaboração entre as partes envolvidas no processo produtivo e a diminuição dos custos de produção (WOMACK; JONES; ROOS, 1992).

Da Produção Enxuta, Womack e Jones (1998) definiram o conceito Pensamento Enxuto como "[...] uma forma de especificar valor, alinhar na melhor sequência as ações que criam valor, realizar essas atividades sem interrupção toda vez que alguém as solicita e realiza-las de forma cada vez mais eficaz" (WOMACK; JONES, 1998, p. 3) e abstraíram seus cinco princípios básicos: (1) Valor; (2) Fluxo de Valor; (3) Fluxo; (4) Puxar (a produção); e (5) Perfeição. Estes princípios emergiram de estudos realizados por Womack e Jones sobre várias empresas em todo o mundo, em vários tipos de indústrias, que tiveram por objetivo a obtenção de respostas sobre como transformar organizações de produção em massa em organizações enxutas, eliminando desperdícios e aumentando a eficiência de seus processos.

O princípio Valor refere-se a um produto específico, bem ou serviço, destinado a atender às necessidades do cliente a um preço e momento específico. 0 Fluxo de Valor consiste nas atividades que são necessárias para a entrega do valor ao cliente. O Fluxo é caracterizado pela realização ininterrupta de todas as atividades do fluxo de valor, sem a ocorrência de desperdícios de tempo e recursos. Puxar (a produção) considera que sempre há a demanda para a realização de uma atividade; na visão completa do processo, o cliente é o responsável por puxar a sua execução, disparando, do fim para o início, as necessidades de trabalho. 0 princípio da Perfeição decorre da prática dos outros princípios que resulta na eliminação de desperdícios, promoção da melhoria contínua e condução da organização em direção à excelência (WOMACK; JONES, 1998).

Godinho Filho e Fernandes (2004), ao realizarem uma revisão bibliográfica sobre a Manufatura Enxuta ${ }^{1}$, se apoiaram em importantes referências sobre o STP e

${ }^{1}$ Manufatura Enxuta (Lean Manufacturing) também é uma denominação para a Produção Enxuta. 
a Manufatura Enxuta para identificar os princípios e os capacitadores que tornam possível a implantação de sistemas enxutos nas empresas, conforme apresentado no Quadro 1.

Quadro 1: Princípios e capacitadores da manufatura enxuta

\begin{tabular}{|c|c|}
\hline Princípios & Capacitadores \\
\hline $\begin{array}{l}\text { Determinar valor para o cliente, } \\
\text { identificando cadeia de valor e } \\
\text { eliminando desperdícios. }\end{array}$ & $\begin{array}{l}\text { - Mapeamento do fluxo de valor; } \\
\text { - Melhoria na relação cliente-fornecedor / redução do número } \\
\text { de fornecedores; } \\
\text { - Recebimento / fornecimento just in time. }\end{array}$ \\
\hline Trabalho em fluxo/simplificar fluxo. & $\begin{array}{l}\text { - Tecnologia de grupo; } \\
\text { - Trabalho em fluxo contínuo (one piece flow) / redução } \\
\text { tamanho de lote; } \\
\text { - Trabalhar de acordo com o takt time / produção } \\
\text { sincronizada; } \\
\text { - Manutenção produtiva total (TPM). }\end{array}$ \\
\hline Produção puxada/Just in time. & $\begin{array}{l}\text { - Recebimento / fornecimento just in time; } \\
\text { - Kanban; } \\
\text { - Redução do tempo de set up. }\end{array}$ \\
\hline Busca da perfeição. & - Kaizen. \\
\hline $\begin{array}{l}\text { Autonomação/qualidade } \\
\text { sigmas. }\end{array}$ & $\begin{array}{l}\text { - Ferramentas de controle da qualidade; } \\
\text { - Zero defeito; } \\
\text { - Ferramentas poka yoke. }\end{array}$ \\
\hline Limpeza, ordem e segurança. & - $5 \mathrm{~S}$. \\
\hline $\begin{array}{l}\text { Desenvolvimento e capacitação de } \\
\text { recursos humanos. }\end{array}$ & $\begin{array}{l}\text { - Empowerment; } \\
\text { - Trabalho em equipes; } \\
\text { - Comprometimento dos funcionários e da alta gerência; } \\
\text { - Trabalhador multi-habilitado / rodízio de funções; } \\
\text { - Treinamento de pessoal. }\end{array}$ \\
\hline Gerenciamento visual. & $\begin{array}{l}\text { - Medidas de performance / balanced scorecard; } \\
\text { - Gráficos de controle visuais. }\end{array}$ \\
\hline $\begin{array}{l}\text { Adaptação de outras áreas da } \\
\text { empresa ao pensamento enxuto. }\end{array}$ & $\begin{array}{l}\text { - Modificação de estrutura financeira/custos; } \\
\text { - Ferramentas para projeto enxuto (DFMA, etc.). }\end{array}$ \\
\hline
\end{tabular}

Fonte: Adaptado de Godinho Filho e Fernandes (2004).

Dessa forma, Godinho Filho e Fernandes (2004) contribuíram com uma identificação mais detalhada de características que constituem o Pensamento Enxuto ao relacionarem os capacitadores que auxiliam na aplicação de cada um de seus princípios. No âmbito do processo de gestão do conhecimento, constatam-se como capacitadores o Empowerment; o trabalho em equipes; trabalhador multihabilitado / rodízio de funções e o treinamento de pessoal.

Em relação ao conhecimento organizacional, Nonaka e Takeuchi (1997) consideram 2 tipos de conhecimento, o tácito e o explícito. Choo (2003) propõe mais um tipo de conhecimento nas organizações, o conhecimento cultural, que diz respeito às estruturas cognitivas e emocionais, valores e crenças que os indivíduos em uma organização utilizam para interpretar e construir a realidade. Nonaka, Toyama e Konno (2000) consideram que o conhecimento é dinâmico, resultante de interações sociais entre indivíduos e organizações, essencialmente relacionado à ação humana e dependente do contexto.

0 conhecimento tácito é difícil de ser formulado e comunicado, pois é de natureza pessoal e inerente a um contexto específico (NONAKA; TAKEUCHI, 1997). Para Choo (2003), o conhecimento tácito refere-se às habilidades de um indivíduo ou aos conjuntos de práticas compartilhados em uma equipe de trabalho, pode ser ensinado e compartilhado informalmente por meio de exemplos. Também é indispensável para que ocorra o aprendizado e a inovação nas organizações.

O conhecimento explícito pode ser expresso formalmente e compartilhado de várias formas, como fórmulas, especificações e manuais; e ainda é facilmente 
processado, transmitido e armazenado (NONAKA; TOYAMA; KONNO, 2000).

Nonaka e Takeuchi (1997) consideram que os conhecimentos tácito e explícito são complementares e a interação entre eles compõe o que eles denominam como processo de conversão do conhecimento:

Em nossa visão, contudo, o conhecimento tácito e o conhecimento explícito não são entidades totalmente separadas, e sim mutualmente complementares. Interagem um com o outro e realizam trocas nas atividades criativas dos seres humanos. Nosso modelo dinâmico de criação do conhecimento está ancorado no pressuposto crítico de que o conhecimento humano é criado e expandido através da interação social entre o conhecimento tácito e o conhecimento explícito. Chamamos essa interação de "conversão do conhecimento" (NONAKA; TAKEUCHI, 1997, p. 67).

Assim, Nonaka e Takeuchi (1997) propuseram quatro fases para o processo de conversão do conhecimento, e o denominaram como processo SECI (Socialização, Externalização, Combinação e Internalização), conforme apresentado no Quadro 2.

Quadro 2: Fases do processo de conversão do conhecimento

\begin{tabular}{|c|l|}
\hline \multicolumn{1}{|c|}{ Fase } & \multicolumn{1}{|c|}{ Descrição } \\
\hline Socialização & $\begin{array}{l}\text { Conversão de conhecimento tácito em conhecimento tácito, na medida em que as } \\
\text { experiências dos indivíduos são compartilhadas uns com os outros. }\end{array}$ \\
\hline Externalização & $\begin{array}{l}\text { Conversão do conhecimento tácito em conhecimento explícito, resultante da } \\
\text { interação e do compartilhamento de informações e experiências entre os } \\
\text { indivíduos. Dessa interação, o conhecimento é explicitado principalmente na forma } \\
\text { de metáforas, analogias e modelos. A externalização é o ponto chave para a criação } \\
\text { do conhecimento, pois faz emergir conceitos novos e explícitos com base nos } \\
\text { conhecimentos tácitos dos indivíduos. }\end{array}$ \\
\hline Combinação & $\begin{array}{l}\text { Conversão de conhecimentos explícitos em novos conhecimentos explícitos, por } \\
\text { meio de um processo de sistematização de conceitos e combinação de diferentes } \\
\text { grupos de conhecimento explícito. Nesse processo, os indivíduos trocam e } \\
\text { combinam conhecimentos já codificados de tal forma que a reconfiguração das } \\
\text { informações existentes leva à criação de novos conhecimentos. }\end{array}$ \\
\hline Internalização & $\begin{array}{l}\text { Incorporação do conhecimento explícito em conhecimento tácito, que é } \\
\text { possibilitada pela repetição de tarefas em um contexto de aprendizagem e } \\
\text { socialização, iniciando assim um novo ciclo de criação do conhecimento. As } \\
\text { experiências adquiridas nos processos de socialização, externalização e } \\
\text { combinação tornam-se ativos de alto valor quando são internalizadas pelos } \\
\text { indivíduos. }\end{array}$ \\
\hline
\end{tabular}

Fonte: Adaptado de Nonaka e Takeuchi (1997).

Von Krogh, Ichijo e Nonaka (2001) denominam o processo de promoção da criação de conhecimento como capacitação para o conhecimento e o definem como "o conjunto geral de atividades organizacionais que afetam de maneira positiva a criação do conhecimento" (VON KROGH; ICHIJO; NONAKA, 2001, p. 12). Ainda, identificam cinco capacitadores do conhecimento: (1) Instilar a visão do conhecimento; (2) Gerenciar as conversas; (3) Mobilizar os ativistas do conhecimento; (4) Contexto adequado; e (5) Globalizar o conhecimento.

Dentre esses capacitadores, a criação do contexto adequado implica em desenvolver "um espaço compartilhado que fomente novos relacionamentos" (VON KROGH; ICHIJO; NONAKA, 2001, p. 16). Esse espaço, denominado como contexto capacitante ou $b a$ - expressão japonesa que significa 'lugar', é essencial para a eficácia da criação de conhecimento e pode ser físico, virtual e mental, ou uma combinação desses tipos de espaço (VON KROGH; ICHIJO; NONAKA, 2001). Em síntese, o contexto capacitante é "... o lugar onde se compartilha, se cria e se utiliza conhecimento. [...] Acima de tudo, trata-se de uma rede de interações, determinada pela solicitude e pela confiança dos participantes." (VON KROGH; ICHIJO; NONAKA, 2001, p. 66). 0 Quadro 3 apresenta os fatores facilitadores para a promoção da solicitude no ambiente 
organizacional.

Quadro 3: Fatores facilitadores para a solicitude nas organizações

\begin{tabular}{|c|c|c|}
\hline Ação & Dimensão & Fatores facilitadores \\
\hline \multirow{5}{*}{$\begin{array}{l}\text { Promover a } \\
\text { solicitude nas } \\
\text { organizações }\end{array}$} & Confiança mútua & $\begin{array}{l}\text { - Compreensão do valor das experiências e histórias de outras } \\
\text { pessoas; } \\
\text { - Disseminação do valor de confiar nas pessoas. }\end{array}$ \\
\hline & Empatia ativa & $\begin{array}{l}\text { - Proatividade das pessoas; } \\
\text { - Compreensão da situação e das necessidades de cada uma das } \\
\text { pessoas; } \\
\text { - Promoção de diálogos interativos; } \\
\text { - Reconhecimento e respeito com questões emocionais das } \\
\text { pessoas; } \\
\text { - Bons relacionamentos pessoais; } \\
\text { - Incentivos para a cooperação, compartilhamento, lealdade e } \\
\text { criatividade entre as pessoas. }\end{array}$ \\
\hline & Acesso à ajuda & $\begin{array}{l}\text { - Proximidade das pessoas para a obtenção de ajuda ou auxílio; } \\
\text { - Fomento para o compartilhamento e a distribuição do } \\
\text { - } \text { conhecimento tácito; } \\
\text { - Mecanismos de compartilhamento de conhecimentos, novas } \\
\text { - Existêncianceitos entre as pessoas; } \\
\text { - Valorização da aprendizagem e da experiência; } \\
\text { - Formação de microcomunidades de conhecimento. }\end{array}$ \\
\hline & $\begin{array}{l}\text { Leniência nos } \\
\text { julgamentos }\end{array}$ & $\begin{array}{l}\text { - Incentivo a atitudes lenientes entre as pessoas; } \\
\text { - Oportunidades de experimentação que levem ao crescimento } \\
\text { das pessoas e à criação de conhecimento; } \\
\text { - Valorização de críticas construtivas; } \\
\text { - Processo de aprendizagem com tolerância e compreensão dos } \\
\text { erros. }\end{array}$ \\
\hline & Coragem & $\begin{array}{l}\text { - Disposição das pessoas para se exporem em situações de } \\
\text { experimentação e avaliação; } \\
\text { - Práticas de feedback construtivas. }\end{array}$ \\
\hline
\end{tabular}

Fonte: Adaptado de Von Krogh, Ichijo e Nonaka (2001).

Assim, o contexto adequado para a criação de conhecimento é o espaço que possibilita aos indivíduos uma interação social marcada pela confiança e solicitude, no qual os fatores facilitadores propiciam as condições favoráveis para a criação de conhecimento nas organizações.

\section{METODOLOGIA}

Para a realização desta revisão de literatura foram adotadas recomendações de Moher et al. (2009), pertinentes ao método PRISMA (Preferred Reporting Items for Systematic Reviews).

A questão que orientou o desenvolvimento da pesquisa foi a seguinte: Quais as práticas do Pensamento Enxuto que são fatores facilitadores para a criação e disseminação de conhecimento nas organizações?

Conforme Figura 1, o processo de definição do corpus de análise constituiu-se de 5 etapas: (1) Pesquisa Inicial; (2) Eliminação de itens duplicados; (3) Eliminação de itens a partir da leitura de títulos e resumos; (4) Eliminação de itens a partir da leitura de textos completos; e (5) Análise dos artigos selecionados. 


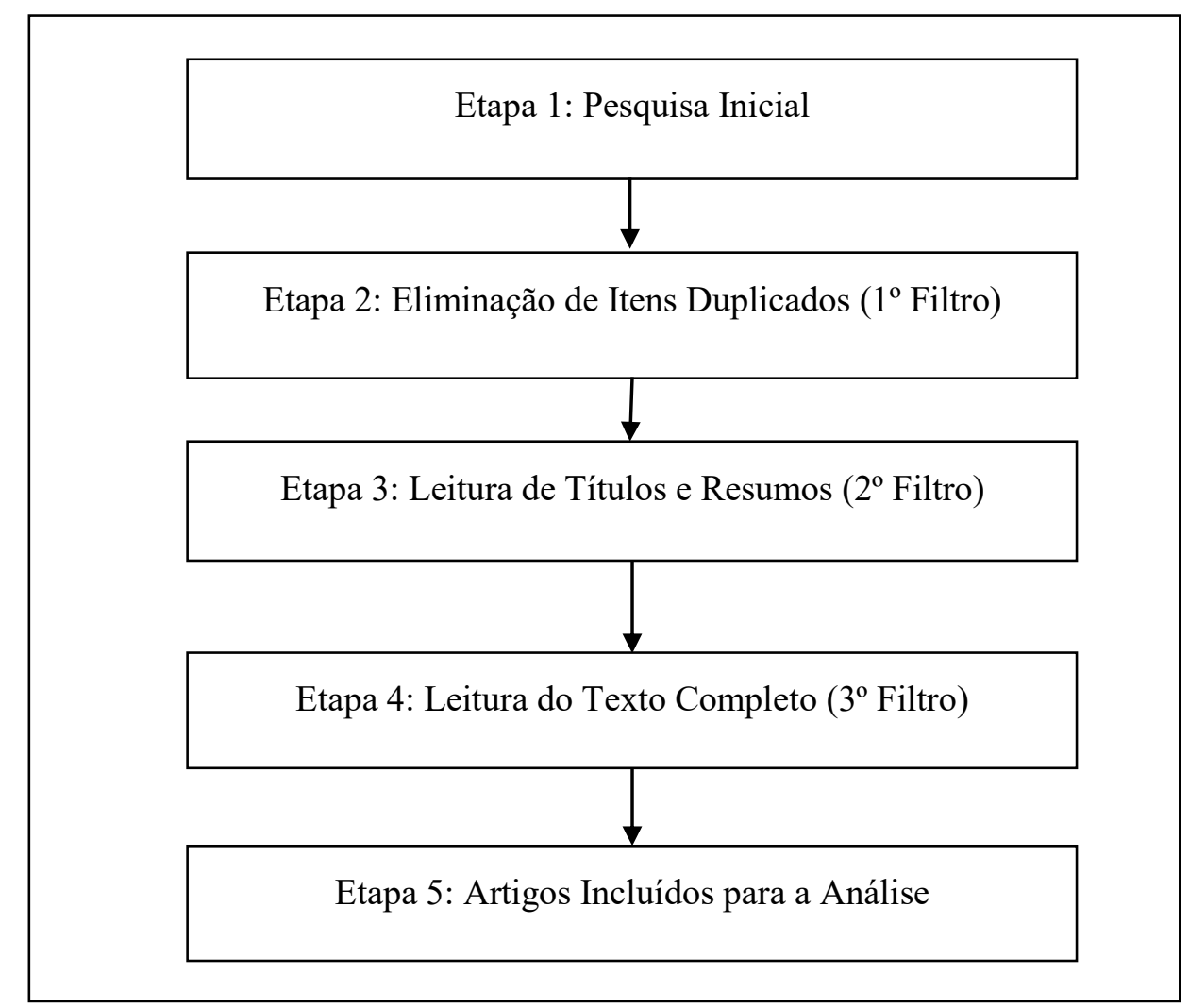

Figura 1: Fluxo do processo de seleção dos artigos Fonte: Adaptado de Moher et al. (2009).

Na estratégia de busca considerou-se os seguintes critérios: a) Bases de Dados: Scopus, Lista-Ebsco, Emerald, Web of Science e Science Direct; b) Data de Publicação: entre os anos de 2007 a 2016; c) Campos: Título, Resumo e Palavras-chave; e d) Expressão: ("lean thinking" OR "lean production" OR "lean manufacturing") AND ("knowledge management" OR "knowledge creation").

A pesquisa inicial nas bases de dados escolhidas foi realizada a partir da aplicação de uma estratégia de busca que associou variações dos termos Lean (Lean Thinking, Lean Production ou Lean Manufacturing) e Knowledge (Knowledge Management ou Knowledge Creation). Essa estratégia foi definida tendo em vista a necessidade de encontrar estudos da temática Lean que também tratassem da gestão ou criação de conhecimento.

Na segunda etapa aplicou-se o $1^{\circ}$ filtro para eliminar os documentos duplicados. Em sequência, na terceira etapa, o 2 o filtro consistiu na leitura dos títulos e resumos dos trabalhos para selecionar os documentos que potencialmente continham informações relevantes para a discussão da questão de pesquisa deste estudo.

Na quarta e quinta etapa foi realizada uma análise pormenorizada dos textos disponíveis, com o objetivo de identificar as práticas mais relevantes no contexto de aplicação do Pensamento Enxuto que favorecem a criação de conhecimento organizacional.

\section{RESULTADOS E DISCUSSÃO}

$\mathrm{Na}$ pesquisa inicial foram identificados 91 estudos, dos quais 15 foram eliminados por estarem duplicados. Após a leitura dos títulos e resumos dos trabalhos restaram 23 estudos que foram selecionados para a análise detalhada do texto completo, por conterem informações relevantes para a discussão da questão de pesquisa deste estudo. Esses procedimentos estão sintetizados na Figura 2. 


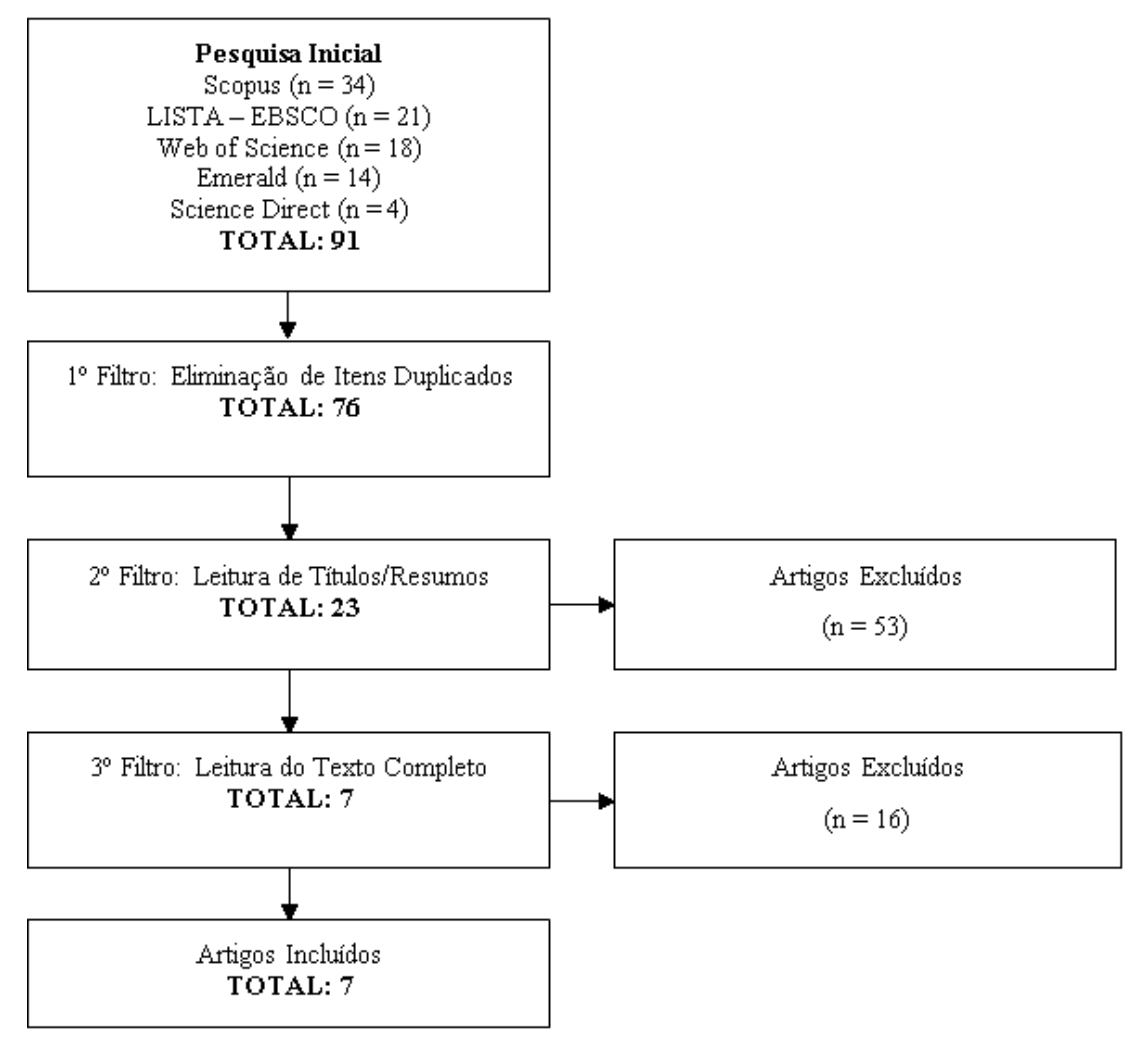

Figura 2: Síntese do processo de seleção dos artigos Fonte: Adaptado de Moher et al. (2009).

Desses 23 estudos selecionados com a aplicação do $2^{\circ}$ filtro, não foi possível o acesso ao texto completo de 16 trabalhos, pois a comunidade acadêmica da Universidade Federal do Paraná - UFPR, da qual os autores deste artigo fazem parte, não possui acesso completo e irrestrito aos periódicos científicos em que esses trabalhos foram publicados, tanto pelo Portal de Periódicos da Capes como pelas bases de dados restritas disponibilizadas internamente pelo Sistema de Bibliotecas da UFPR. Assim, a leitura do texto completo prosseguiu em sete artigos para a análise e discussão da temática proposta. A relação dos artigos incluídos na análise está apresentada no Apêndice A.

No Quadro 4 estão apresentadas as sete práticas do Pensamento Enxuto que foram identificadas na literatura como facilitadoras para a criação e disseminação de conhecimento organizacional. 
Quadro 4: Práticas facilitadoras do pensamento enxuto para a criação e disseminação de conhecimento organizacional

\begin{tabular}{|c|c|c|}
\hline Práticas & Constatações & Autores \\
\hline \multirow{2}{*}{$\begin{array}{c}\text { Estrutura } \\
\text { Organizacional }\end{array}$} & $\begin{array}{l}\text { A estrutura organizacional proveniente de sistemas Lean é uma das } \\
\text { características que influência positivamente a atividade de } \\
\text { compartilhamento de conhecimento. }\end{array}$ & $\begin{array}{l}\text { Cavaliere, Lombardi } \\
\quad \text { e Sarti (2012) }\end{array}$ \\
\hline & $\begin{array}{l}\text { A estrutura organizacional e a gestão do conhecimento estão } \\
\text { fortemente inter-relacionadas nos sistemas de produção enxuta, devido } \\
\text { ao fato de que o conhecimento deve ser transferido entre as diferentes } \\
\text { funções da organização. }\end{array}$ & $\begin{array}{l}\text { Dombrowski, Mielke } \\
\text { e Engel (2012) }\end{array}$ \\
\hline Autonomia & $\begin{array}{l}\text { Fator relevante para os empregados compartilharem conhecimentos, } \\
\text { sendo que quanto maior o grau de autonomia, maior a possibilidade de } \\
\text { os empregados adotarem comportamentos que facilitam o } \\
\text { compartilhamento de conhecimentos. }\end{array}$ & $\begin{array}{l}\text { Cavaliere, Lombardi } \\
\text { e Sarti (2012) }\end{array}$ \\
\hline \multirow{3}{*}{$\begin{array}{l}\text { Mecanismos de } \\
\text { Integração }\end{array}$} & $\begin{array}{l}\text { Instrumentos que ajudam a estabelecer canais de comunicação entre as } \\
\text { diversas áreas, facilitando a aquisição e disseminação de conhecimento. }\end{array}$ & $\begin{array}{l}\text { Cavaliere, Lombardi } \\
\quad \text { e Sarti (2012) }\end{array}$ \\
\hline & $\begin{array}{l}\text { A utilização de ferramentas visuais nos sistemas Lean facilitam os } \\
\text { processos de combinação e internalização de conhecimentos. }\end{array}$ & Tyagi et al. (2015) \\
\hline & $\begin{array}{l}\text { Promovem a transferência de conhecimento } \\
\text { compartilhamento de informações. }\end{array}$ & $\begin{array}{l}\text { Wangwacharakul et } \\
\text { al. (2014) }\end{array}$ \\
\hline $\begin{array}{l}\text { Replicação de } \\
\text { Conhecimento }\end{array}$ & $\begin{array}{l}\text { A replicação de conhecimento baseada em princípios torna menos } \\
\text { engessado o processo de transferência de conhecimento e possibilita } \\
\text { compreender a melhor forma de aplicar a lógica Lean, analisar } \\
\text { problemas e prever soluções, tornando o processo de implantação de } \\
\text { sistemas Lean mais rápido e satisfatório. }\end{array}$ & $\begin{array}{l}\text { Secchi e Camuffo } \\
\qquad(2016)\end{array}$ \\
\hline $\begin{array}{l}\text { Ambidesteridade } \\
\text { Contextual }\end{array}$ & $\begin{array}{l}\text { A ambidesteridade contextual em sistemas enxutos torna os processos } \\
\text { mais eficazes e eficientes. A ambidesteridade contextual diz respeito a } \\
\text { coexistência das seguintes práticas: exploration de conhecimento } \\
\text { (introdução de práticas inovativas) e exploitation de conhecimento } \\
\text { (gerenciamento de operações de rotina). }\end{array}$ & $\begin{array}{l}\text { Secchi e Camuffo } \\
\qquad(2016)\end{array}$ \\
\hline Aprendizagem & $\begin{array}{l}\text { A aprendizagem on-the-job, os workshops de treinamento e a rotação } \\
\text { nos postos de trabalho estimulam o desenvolvimento do conhecimento } \\
\text { nas organizações. }\end{array}$ & $\begin{array}{l}\text { Wangwacharakul et } \\
\text { al. (2014) }\end{array}$ \\
\hline \multirow{4}{*}{ Ferramentas Lean } & $\begin{array}{l}\text { O Mapeamento de Fluxo de Valor (MFV) possibilita examinar e } \\
\text { documentar aspectos do conhecimento em cada etapa do processo, } \\
\text { contribuindo para a identificação e visualização do conhecimento. }\end{array}$ & Kern et al. (2011) \\
\hline & $\begin{array}{l}\text { O Mapeamento de Fluxo de Valor (MFV) possibilita o desenvolvimento } \\
\text { dos fluxos de conhecimento nas organizações. }\end{array}$ & $\begin{array}{l}\text { Zhao, Rasovska e } \\
\text { Rose (2016) }\end{array}$ \\
\hline & $\begin{array}{l}\text { A Análise de Causa-Raiz ( } 5 \text { porquês) facilita todas as etapas do processo } \\
\text { de conversão de conhecimento. }\end{array}$ & Tyagi et al. (2015) \\
\hline & $\begin{array}{l}\text { A Análise de Causa-Raiz (5 porquês) contribui para o desenvolvimento } \\
\text { dos fluxos de conhecimento nas organizações. }\end{array}$ & $\begin{array}{l}\text { Zhao, Rasovska e } \\
\text { Rose (2016) }\end{array}$ \\
\hline
\end{tabular}

Fonte: Elaborado pelos autores (2017).

Em relação à Estrutura Organizacional, Dombrowski, Mielke e Engel (2012) consideram que nos sistemas de produção enxuta prevalece uma forte relação entre a estrutura organizacional e a gestão do conhecimento, pela razão de que o conhecimento deve ser transferido entre as diferentes funções da organização. A implantação de sistemas de produção enxuta se caracteriza por uma transição fundamental dos 
princípios, métodos e ferramentas da organização. Dessa forma, a adaptação das operações em um sistema de produção enxuta somente pode ser alcançada quando a informação sobre princípios, métodos e ferramentas é corretamente compreendida em todas as áreas da organização.

De acordo com Cavaliere, Lombardi e Sarti (2012), que analisaram o processo de compartilhamento de conhecimento em empresas italianas, a estrutura organizacional proveniente de sistemas Lean é uma das características que influencia positivamente a atividade de compartilhamento de conhecimento. Esses autores consideram os princípios Lean, em relação à capacidade da empresa de valorizar as equipes de trabalho multiqualificadas, na implementação de práticas de gestão de conhecimento e de uma cultura de melhoria contínua.

Em empresas com estrutura organizacional mais flexível, menos hierárquica, com espaço para a criatividade e inovação, torna-se mais provável estabelecer uma cultura de compartilhamento de conhecimento e incrementar os fluxos de conhecimento e de aprendizagem. Assim, ideias e opiniões são valorizadas, empregados possuem autonomia e todos são estimulados a executar o melhor de suas habilidades (CAVALIERI; LOMBARDI; SARTI, 2012).

A Autonomia no trabalho é uma das variáveis organizacionais Lean, identificada por Cavaliere, Lombardi e Sarti (2012), que também está positivamente relacionada com a atividade de compartilhamento de conhecimento, pois é um fator relevante para os empregados compartilharem conhecimentos, sendo que quanto maior o grau de autonomia, maior a possibilidade de os empregados adotarem comportamentos que facilitam o compartilhamento de conhecimentos (CAVALIERI; LOMBARDI; SARTI, 2012). Para Secchi e Camuffo (2016), a autonomia está associada com o grau de descentralização da tomada de decisão e contribui positivamente para a maior eficiência e eficácia dos processos de implantação de sistemas Lean.

Os Mecanismos de Integração consistem em instrumentos que favorecem o compartilhamento de conhecimento. São os instrumentos existentes nas organizações que ajudam a estabelecer canais de comunicação entre as diversas áreas, facilitando a aquisição e disseminação de conhecimento (CAVALIERI; LOMBARDI; SARTI, 2012). Nesse sentido, a utilização de ferramentas visuais nos sistemas Lean facilitam os processos de combinação e internalização de conhecimentos (TYAGI et al, 2015) e, também promovem a transferência de conhecimento por meio do compartilhamento de informações (WANGWACHARAKUL et al., 2014).

Conforme Secchi e Camuffo (2016), a Replicação de Conhecimento baseada em princípios torna menos engessado o processo de transferência de conhecimento, quando comparada com uma estratégia baseada no uso de modelos pré-definidos e alto grau de codificação de conhecimento. Essa abordagem possibilita uma melhor compreensão de como aplicar a lógica Lean em contextos específicos, compreender problemas e prever soluções, tornando o processo de implantação de sistemas Lean mais rápidos e satisfatórios. Esses autores apontam que tal evidência é consistente com pesquisas recentes sobre sistemas Lean, que demonstram:

[...] a complexidade [da implantação de sistemas de produção enxuta] requer uma conceituação que vai além da ideia de um sistema de práticas para promover a ideia de um sistema de gerenciamento que inclua as rotinas organizacionais, rotinas de aprendizagem, cultura organizacional e liderança" (SECCHI; CAMUFFO, 2016, p. 78, tradução nossa).

Assim, Secchi e Camuffo (2016) consideram os sistemas Lean como complexos e mais orientados para uma abordagem dinâmica de aprendizagem e de criação de conhecimento, ao invés de uma simples replicação de práticas. Por essa razão propuseram que "quanto menor o grau de codificação de conhecimento, maior será a eficiência e a eficácia do processo de implantação de sistemas enxutos" (SECCHI; 
CAMUFFO, 2016, p. 79, tradução nossa).

Em relação à Ambidesteridade Contextual, Secchi e Camuffo (2016) argumentam, com base na investigação que realizaram sobre a implantação de sistemas de produção enxuta em unidades de empresas multinacionais, que a predominância da ambidesteridade ${ }^{2}$ contextual é um dos fatores que torna esses processos mais eficazes e eficientes. Segundo esses autores, a ambidesteridade contextual diz respeito a coexistência das seguintes práticas: exploration de conhecimento (introdução de práticas inovativas) e exploitation de conhecimento (gerenciamento de operações de rotina). Dentre os casos analisados, as unidades que buscaram simultaneamente a exploração das capacidades operacionais existentes e a exploração de novas capacidades operacionais foram mais eficientes e eficazes no processo de implantação de sistemas de produção enxuta.

Dentre as possibilidades de Aprendizagem no contexto de aplicação dos princípios do Pensamento Enxuto, Wangwacharakul et al. (2014) consideram o tipo de aprendizagem on-the-job, ou 'aprender fazendo', os workshops de treinamento e a rotação nos postos de trabalho como fatores que estimulam o desenvolvimento do conhecimento nas organizações. Conforme Tyagi et al. (2015), o Pensamento Enxuto incentiva o líder a treinar pacientemente o aprendiz, que 'aprende fazendo' sob sua supervisão direta. Esse tipo de aprendizagem requer intensamente das pessoas a vontade de comunicar e interagir nas atividades do dia-a-dia. A prática dessa abordagem, na visão de Tyagi et al. (2015), é caracterizada como um processo de socialização do conhecimento, com trocas de conhecimentos tácitos, e pode influenciar o modo como a organização permite que seus empregados compartilhem suas experiências, intuições, observações e imitações. Outro modo de socialização do conhecimento citado por esses autores são os encontros informais, visto que o incentivo às atividades sociais fora do local de trabalho capacita as equipes a obterem novos insights.

O Mapeamento de Fluxo de Valor (MFV) é uma Ferramenta Lean que possibilita examinar e documentar aspectos do conhecimento em cada etapa do processo, contribuindo para a identificação e visualização do conhecimento (KERN et al, 2011) e para o desenvolvimento dos fluxos de conhecimento nas organizações (ZHAO; RASOVSKA; ROSE, 2016). Assim, a criação de conhecimento pode ser reunida em detalhes e documentada em termos de conteúdo e tipo de conhecimento, facilitando a seleção de processos, métodos e ferramentas para a gestão de conhecimento (KERN et al, 2011).

Além do MFV, a Análise de Causa-Raiz (5 Porquês) é uma ferramenta que facilita todas as etapas do processo de conversão de conhecimento SECI, de Nonaka e Takeuchi (1997), conforme argumentado por Tyagi et al (2015), e contribui para o desenvolvimento dos fluxos de conhecimento nas organizações (ZHAO; RASOVSKA; ROSE, 2016).

\section{CONSIDERAÇÕES FINAIS}

Esta revisão de literatura evidenciou a existência de práticas do Pensamento Enxuto, em suas aplicações, que facilitam a criação e disseminação de conhecimento organizacional, e, numa visão mais ampla, o processo de gestão de conhecimento. Desta forma, constata-se que o objetivo proposto para o estudo foi alcançado. Em síntese, as características ou ferramentas mais relevantes de cada uma das práticas identificadas são:

2 Conforme Nieto-Rodriguez (2014), a definição mais aceita para ambidesteridade é um equilíbrio entre exploration e exploitation, ou seja, quando as organizações são capazes de explorar as suas competências existentes (exploitation) ao mesmo tempo em que exploram novas oportunidades (exploration). 
1. Estrutura organizacional: flexibilidade, baixa hierarquia, trabalho em equipe, trabalhadores multiqualificados, cultura de melhoria contínua, criatividade e inovação.

2. Autonomia: descentralização da tomada de decisão e autonomia dos trabalhadores.

3. Mecanismos de integração: canais de comunicação e ferramentas visuais.

4. Replicação de conhecimento: abordagem por princípios.

5. Ambidesteridade contextual: Exploration e Exploitation.

6. Aprendizagem: treinamentos on-the-job, workshops de treinamento, rotação nos postos de trabalho, interação, comunicação e encontros informais.

7. Ferramentas Lean: Mapeamento de Fluxo de Valor e Análise de Causa-Raiz.

A aplicação dos princípios do Pensamento Enxuto, via de regra, possibilita ganhos de eficiência e produtividade, que são auferidos pela eliminação de desperdícios nos processos de trabalho. Entretanto, com base nas constatações deste estudo, verificase também que o Pensamento Enxuto promove o desenvolvimento de um contexto adequado para a gestão do conhecimento organizacional As características e ferramentas das sete práticas identificadas tornam possíveis todas as fases do processo de conversão de conhecimento (processo SECI) e estimulam redes de interações nas quais o conhecimento é criado, compartilhado e utilizado pelas pessoas nos ambientes organizacionais.

Uma limitação deste estudo se deve ao fato de que não foi possível acessar o texto completo de 16 artigos, de um total de 23 documentos selecionados. Com isso, os resultados encontrados retratam parcialmente o conjunto de práticas do Pensamento Enxuto que facilitam a criação e disseminação de conhecimento organizacional.

Para trabalhos futuros, sugere-se a complementação deste estudo por meio da análise de outras pesquisas sobre o Pensamento Enxuto que, por ora, não fizeram parte do corpus de análise, de modo a ampliar a discussão e identificar mais fatores do Pensamento Enxuto que facilitam o processo de criação e disseminação do conhecimento organizacional.

\section{REFERÊNCIAS}

CAVALCANTE, L. F. B.; VALENTIM, M. L. P. Informação e conhecimento no contexto dos ambientes organizacionais. In: VALENTIM, M. L. P. (Org.). Gestão, mediação e uso da informação. São Paulo: Cultura Acadêmica, 2010.

CAVALIERE, V. F.; LOMBARDI, S.; SARTI, D. Enabling Employees' Knowledge Sharing Through Organizational and Individual Factors: Evidence From Italian Firms. Proceedings of the European Conference on Knowledge Management, v. 1, p. 179-188, jan. 2012.

CHOO, C. W. A organização do conhecimento: como as organizações usam a informação para criar significado, construir conhecimento e tomar decisões. São Paulo: Editora Senac São Paulo, 2003. Tradução de: Eliana Rocha.

DOMBROWSKI, U.; MIELKE, T.; ENGEL, C. Knowledge Management in Lean Production Systems. Procedia CIRP, v. 3, p. 436-441, 2012.

GODINHO FILHO, M.; FERNANDES, F. C. F. Manufatura Enxuta: uma revisão que classifica e analisa os trabalhos apontando perspectivas de pesquisas futuras. Gestão \& Produção, v. 11, n. 1, p. 1-19, 2004.

KERN, E. M. et al. Integration of knowledge into the value stream mapping method - benefits, challenges, solution statement. Proceedings of the European Conference on Knowledge Management, ECKM. Anais... 2011. 
MOHER, D. et al. Preferred Reporting Items for Systematic Reviews and Meta-Analyses: The PRISMA Statement. Physical Therapy, v. 89, n. 9, p. 873-880, 2009.

NIETO-RODRIGUEZ, A. Organisational ambidexterity. 2014. Disponível em: $<$ https://www.london.edu/facultyandresearch/lbsr/organisationalambidexterity\#.WIOpbP krKUl>. Acesso em: 18 jan. 2017.

NONAKA, I.; TAKEUCHI, H. Criação de conhecimento na empresa: como as empresas japonesas geram a dinâmica da inovação. Rio de Janeiro: Elsevier, 1997.

; TOYAMA, R.; KONNO, N. SECI, Ba and Leadership: a Unified Model of Dynamic Knowledge Creation. Long Range Planning, v. 33, n. 1, p. 5-34, 2000.

SECCHI, R.; CAMUFFO, A. Rolling out lean production systems: a knowledge-based perspective. International Journal of Operations \& Production Management, v. 36, n. 1, p. 61-85, 4 jan. 2016.

TYAGI, S. et al. Lean tools and methods to support efficient knowledge creation.

International Journal of Information Management, v. 35, n. 2, p. 204-214. 2015.

VON KROGH, G.; ICHIJO, K.; NONAKA, I. Facilitando a criação do conhecimento: reinventando a empresa com o poder da inovação. Rio de Janeiro: Campus, 2001.

WANGWACHARAKUL, P. et al. Cultural aspects when implementing lean production and lean product development -experiences from a Swedish perspective. Quality Innovation Prosperity, v. 18, n. 1, 2014.

WOMACK, J. P.; JONES, D. T. A mentalidade enxuta nas empresas. 4 ed. Rio de Janeiro: Campus, 1998.

; JONES, D. T.; ROOS, D. A máquina que mudou o mundo. 5 ed. Rio de Janeiro:

Campus, 1992.

ZHAO, P.; RASOVSKA, I.; ROSE, B. Integrating Lean perspectives and Knowledge Management in Services: application to the service department of a CNC manufacturer. IFAC-PapersOnLine, v. 49, n. 12, p. 77-82, 2016. 
APÊNDICE A - RELAÇÃO DE ARTIGOS ANALISADOS

\begin{tabular}{|c|c|c|c|}
\hline Ano & Autores & Título & Descrição do Estudo \\
\hline 2011 & Kern et al. & $\begin{array}{l}\text { Integration of knowledge } \\
\text { into the value stream } \\
\text { mapping method - } \\
\text { benefits, challenges, } \\
\text { solution statement }\end{array}$ & $\begin{array}{l}\text { Apresentação de um método para } \\
\text { conduzir a gestão de conhecimento em } \\
\text { conjunto com o mapeamento de fluxo de } \\
\text { valor. }\end{array}$ \\
\hline 2012 & $\begin{array}{c}\text { Cavaliere, } \\
\text { Lombardi e Sarti }\end{array}$ & $\begin{array}{lr}\text { Enabling } & \text { Employees' } \\
\text { Knowledge } & \text { Sharing } \\
\text { Through Organizational } \\
\text { and Individual Factors: } \\
\text { Evidence From } & \text { Italian } \\
\text { Firms. } & \end{array}$ & $\begin{array}{l}\text { Investigação da relação entre os } \\
\text { capacitadores de compartilhamento de } \\
\text { conhecimento e processos de } \\
\text { compartilhamento de conhecimento por } \\
\text { meio de uma análise exploratória, com } \\
\text { dados coletados em uma pesquisa } \\
\text { realizada com uma amostra de } \\
318 \text { trabalhadores do conhecimento de } \\
14 \text { empresas intensivas em } \\
\text { conhecimento situadas na Toscânia } \\
\text { (Itália) e que operam em mercados } \\
\text { internacionais. }\end{array}$ \\
\hline 2012 & $\begin{array}{l}\text { Dombrowski, } \\
\text { Mielke e Engel }\end{array}$ & $\begin{array}{lr}\text { Knowledge } & \text { Management } \\
\text { in Lean } & \text { Production } \\
\text { Systems } & \end{array}$ & $\begin{array}{l}\text { Análise de como o conhecimento e os } \\
\text { fluxos de conhecimento podem ser } \\
\text { descritos na implementação de sistemas } \\
\text { de produção enxuta. }\end{array}$ \\
\hline 2014 & $\begin{array}{c}\text { Wangwacharakul } \\
\text { et al. }\end{array}$ & $\begin{array}{l}\text { Cultural aspects when } \\
\text { implementing lean } \\
\text { production and lean } \\
\text { product development - } \\
\text { experiences from a } \\
\text { Swedish perspective }\end{array}$ & $\begin{array}{l}\text { Identificação de aspectos culturais que } \\
\text { influenciam a implementação da } \\
\text { produção enxuta e o desenvolvimento } \\
\text { de produto enxuto em empresas suecas. }\end{array}$ \\
\hline 2015 & Tyagi et al. & $\begin{array}{l}\text { Lean tools and methods to } \\
\text { support efficient } \\
\text { knowledge creation }\end{array}$ & $\begin{array}{l}\text { Discussão da implementação de dez } \\
\text { ferramentas e métodos Lean no modelo } \\
\text { de conhecimento dinâmico integrado, } \\
\text { para aumentar a eficiência da criação de } \\
\text { conhecimento em um domínio de } \\
\text { desenvolvimento de produto. }\end{array}$ \\
\hline 2016 & Secchi e Camuffo & $\begin{array}{l}\text { Rolling out lean } \\
\text { production systems: a } \\
\text { knowledge-based } \\
\text { perspective }\end{array}$ & $\begin{array}{l}\text { Investigação da implantação de sistemas } \\
\text { de produção enxuta em empresas } \\
\text { multinacionais, a partir da visão da } \\
\text { firma baseada no conhecimento. }\end{array}$ \\
\hline 2016 & $\begin{array}{c}\text { Zhao, Rasovska e } \\
\text { Rose }\end{array}$ & $\begin{array}{l}\text { Integrating } \quad \text { Lean } \\
\text { perspectives and } \\
\text { Knowledge Management } \\
\text { in Services: application to } \\
\text { the service department of } \\
\text { a CNC manufacturer }\end{array}$ & $\begin{array}{l}\text { Integração da abordagem Lean em } \\
\text { serviços com uma perspectiva de gestão } \\
\text { do conhecimento. }\end{array}$ \\
\hline
\end{tabular}

